\title{
Immunocompromised Children with Severe Adenoviral Respiratory Infection
}

\author{
Joanna C. Tylka, ${ }^{1}$ Michael C. McCrory, ${ }^{2}$ Shira J. Gertz, \\ Jason W. Custer, ${ }^{4}$ and Michael C. Spaeder ${ }^{5}$ \\ ${ }^{1}$ Department of Pediatrics, Section of Pediatric Critical Care, Rush University Medical Center, Chicago, Il 60612, USA \\ ${ }^{2}$ Department of Anesthesiology, Section of Pediatric Critical Care, Wake Forest School of Medicine, Winston-Salem, NC 27157, USA \\ ${ }^{3}$ Department of Pediatrics, Hackensack University Medical Center, Hackensack, NJ 07601, USA \\ ${ }^{4}$ Division of Pediatric Critical Care Medicine, University of Maryland Medical Center, Baltimore, MD 20201, USA \\ ${ }^{5}$ Division of Pediatric Critical Care, University of Virginia Children's Hospital, Charlottesville, VA 22908, USA
}

Correspondence should be addressed to Joanna C. Tylka; joanna.tylka@me.com

Received 30 November 2015; Accepted 18 April 2016

Academic Editor: Robert Boots

Copyright (C) 2016 Joanna C. Tylka et al. This is an open access article distributed under the Creative Commons Attribution License, which permits unrestricted use, distribution, and reproduction in any medium, provided the original work is properly cited.

\begin{abstract}
Purpose. To investigate the impact of severe respiratory adenoviral infection on morbidity and case fatality in immunocompromised children. Methods. Combined retrospective-prospective cohort study of patients admitted to the intensive care unit (ICU) in four children's hospitals with severe adenoviral respiratory infection and an immunocompromised state between August 2009 and October 2013. We performed a secondary case control analysis, matching our cohort 1:1 by age and severity of illness score with immunocompetent patients also with severe respiratory adenoviral infection. Results. Nineteen immunocompromised patients were included in our analysis. Eleven patients (58\%) did not survive to hospital discharge. Case fatality was associated with cause of immunocompromised state $(p=0.015)$, multiple organ dysfunction syndrome $(p=0.001)$, requirement of renal replacement therapy $(p=0.01)$, ICU admission severity of illness score $(p=0.011)$, and treatment with cidofovir $(p=0.005)$. Immunocompromised patients were more likely than matched controls to have multiple organ dysfunction syndrome $(p=0.01)$, require renal replacement therapy $(p=0.02)$, and not survive to hospital discharge $(p=0.004)$. One year after infection, $43 \%$ of immunocompromised survivors required chronic mechanical ventilator support. Conclusions. There is substantial case fatality as well as short- and long-term morbidity associated with severe adenoviral respiratory infection in immunocompromised children.
\end{abstract}

\section{Introduction}

Adenoviruses are a group of DNA viruses that are estimated to cause $5-10 \%$ of febrile illnesses in infants and children [1]. Infections occur primarily in children less than five years of age and may involve the respiratory tract, conjunctiva, or gastrointestinal tract or become disseminated. Adenovirus is a common cause of acute respiratory infection manifesting as bronchiolitis, wheezing, croup, or pneumonia [2-5]. In immunocompetent hosts most infections are selflimited; however respiratory infection from adenovirus can be severe requiring pediatric intensive care unit admission [2], mechanical ventilation $[2,3,6]$, and extracorporeal life support [7].
Children are at risk for long-term respiratory sequelae from adenoviral respiratory infection. Adenoviral infections have been linked to the development of chronic pneumonia, bronchiectasis, and hyperlucent lung [8-11]. Adenovirus is the most common cause of postinfectious bronchiolitis obliterans, with one study finding that bronchiolitis obliterans developed in $36 \%$ of children with adenoviral acute lower respiratory tract infection [12]. Castro-Rodriguez and colleagues found that $47 \%$ of children who had adenovirus pneumonia developed evidence of bronchiolitis obliterans on chest computed tomography at five-year follow-up [13].

Adenoviruses have emerged as significant opportunistic pathogens in the immunocompromised population, with 
infections ranging from asymptomatic to disseminated disease with fatalities [1]. Mortality rates in immunocompromised populations with adenoviral infection are highly variable, ranging from $2 \%$ to $69 \%$ [14-20], with rates as low as $2 \%$ in prospective screening studies where a large portion of the patients are asymptomatic [14]. Conversely, mortality rates approaching 70\% have been documented in hematopoetic stem cell transplant (HSCT) patients with symptomatic disease $[17,18]$. Prior studies have suggested that adenoviral respiratory infection in the immunocompromised patient may be associated with substantial mortality [15, $16,21]$. We investigated the morbidity and case fatality in immunocompromised children with severe adenoviral respiratory infection as compared to immunocompetent children, as well as the long-term respiratory morbidity in survivors.

\section{Materials and Methods}

We performed a combined retrospective-prospective cohort study of all immunocompromised patients admitted to the intensive care unit (ICU), either pediatric ICU or cardiac ICU, with adenoviral respiratory infection at four tertiary care children's hospitals between August 2009 and October 2013. We also performed a secondary matched case control analysis matching our cohort of immunocompromised patients at a 1:1 ratio on age and severity of illness score with immunocompetent patients with adenoviral respiratory infection. Adenoviral respiratory infection was defined as the identification of adenovirus from a nasopharyngeal or endotracheal specimen by polymerase chain reaction (PCR), direct fluorescent antibody, or shell viral culture with signs and symptoms of respiratory illness (e.g., supplemental oxygen requirement, wheezing, and cough) at the time of specimen collection. Immunocompromised state was defined as immune system dysfunction as a result of medications after solid organ transplant (SOT) or HSCT, neutropenia with absolute neutrophil count (ANC) less than 500 cells $/ \mathrm{mm}^{3}$, congenital immunodeficiency, or human immunodeficiency virus. The institutional review boards at the Children's National Medical Center, Wake Forest Baptist Medical Center, Hackensack University Medical Center, and University of Maryland Medical Center reviewed and approved this study.

Review of clinical, administrative, and laboratory databases was conducted to collect information on patient characteristics and outcomes. Clinical data collected included hospital and ICU length of stay; presence of respiratory failure, multiple organ dysfunction syndrome (MODS), acute respiratory distress syndrome (ARDS), concomitant viral, bacterial, or fungal infection, and other chronic conditions; requirement of mechanical ventilation, renal replacement therapy (RRT), or extracorporeal life support (ECLS); absolute lymphocyte count (ALC) at time of infection; and death before hospital discharge. Case fatality was the primary outcome of interest. Mechanical ventilation use was defined as the initiation of positive pressure ventilation, which includes both invasive modes (e.g., pressure-control ventilation, high frequency oscillatory ventilation) and noninvasive modes (e.g., bilevel positive airway pressure). MODS was established based on the
International Pediatric Sepsis Consensus Conference criteria [22]. The Berlin Definition for Acute Respiratory Distress Syndrome was used to establish a diagnosis of ARDS [23]. The Paediatric Index of Mortality 3 (PIM3) was calculated for each patient at the time of admission to the ICU to establish a severity of illness index and corresponding predicted probability of death [24].

We defined viral coinfection as the identification of other viral agents by PCR, direct fluorescent antibody testing, or shell viral culture testing on the same nasopharyngeal, endotracheal, or bronchoalveolar lavage specimen that identified adenovirus. All three testing modalities could detect, in addition to adenovirus, respiratory syncytial virus, influenza $A$ and $B$, and parainfluenza viruses 1-3. Respiratory viral PCR could additionally detect human metapneumovirus and human rhinoviruses and enteroviruses. We defined bacterial or fungal coinfection as the identification of a bacterial or fungal pathogen in culture from an endotracheal, bronchoalveolar, or sterile blood specimen. Respiratory morbidity was assessed either retrospectively or prospectively at one year following diagnosis of infection. Review of surviving patient's charts was conducted to assess for chronic respiratory diagnoses, requirement of chronic mechanical ventilation, supplemental oxygen requirement, and chronic respiratory medication use. Chronic respiratory diagnosis was defined as any long-term pulmonary diagnosis made by a physician. Requirement of chronic mechanical ventilation or supplemental oxygen was defined as the need for either modality for at least part of every day (e.g., overnight). Chronic respiratory medications were defined as an inhaled medication prescribed to treat or prevent a respiratory disease for at least one month.

In the primary outcomes analysis, continuous variables were compared using Student's $t$-test or Wilcoxon rank sum testing as appropriate and categorical variables were compared using Fisher's Exact testing. In the matched case control analysis, continuous variables were compared using paired $t$-test or Wilcoxon matched pairs signed rank test and categorical variables were compared using McNemar's test. Type-one error was set at 0.05. All calculations were performed using Stata/IC 12.1 (Stata Corporation, College Station, TX).

\section{Results}

There were 19 immunocompromised patients included in our analysis. Seventeen of the patients were diagnosed by respiratory viral PCR, one by direct fluorescent antibody, and one by shell vial culture. Patient characteristics are presented in Table 1 with detailed patient descriptions presented in Table 2. The most common diagnosis was immunocompromised state in the setting of HSCT (47\%). The median age of patients was 3.9 years (interquartile range (IQR) 2.6 to 12.7 ). The median hospital length of stay was 45 days (IQR 17 to 112 ) and median ICU length of stay was 20 days (IQR 7 to 48 ). The median PIM3 predicted mortality for the entire cohort was 5.4\% (IQR $1.1 \%$ to $5.7 \%)$.

Seventeen of the immunocompromised patients (89\%) required mechanical ventilation with a median duration of 
TABLE 1: Characteristics of immunocompromised patients admitted to the intensive care unit with adenoviral respiratory infection.

\begin{tabular}{lc}
\hline Characteristic & Number $(\%)$ \\
\hline Age group & \\
$<1$ year & $2(11)$ \\
1-5 years & $10(53)$ \\
6-10 years & $1(5)$ \\
11-18 years & $6(32)$ \\
Female gender & $11(58)$ \\
Race/ethnicity & \\
Caucasian & $2(11)$ \\
African-American & $10(53)$ \\
Hispanic & $5(26)$ \\
Asian & $2(11)$ \\
Other & $0(0)$ \\
Cause of immunocompromised state & \\
Solid organ transplant & $3(16)$ \\
Hematopoetic stem cell transplant & $9(47)$ \\
Human immunodeficiency virus & $0(0)$ \\
Neutropenia & $3(16)$ \\
Congenital immunodeficiency & $4(21)$ \\
Mechanical ventilation & $17(89)$ \\
Acute respiratory distress syndrome & $7(37)$ \\
Multiple organ dysfunction syndrome & $11(68)$ \\
Renal replacement therapy & $7(37)$ \\
Extracorporeal life support & $1(5)$ \\
Mortality & $11(58)$ \\
\hline
\end{tabular}

Values expressed as $n$ (\%).

ventilation of 12 days (IQR 1-27 days). Seven (37\%) patients met diagnostic criteria for ARDS and 11 (58\%) for MODS. RRT was required for seven patients $(37 \%)$ and one patient (5\%) underwent ECLS. Eleven patients did not survive to discharge yielding a case fatality rate of $58 \%$. Of the eleven nonsurviving patients, eight were immunocompromised due to HSCT (case fatality $89 \%$ ), one due to SOT (case fatality $33 \%$ ), two due to neutropenia (case fatality 67\%), and none due to congenital immunodeficiencies.

Seven patients (37\%) had identification of at least one additional virus on the same specimen that detected adenovirus. All seven patients had detection of human rhino/enterovirus. One patient had detection of respiratory syncytial virus in addition to human rhino-/enterovirus. Three patients $(16 \%)$ had bacterial coinfection and no fungal coinfections were identified.

Antiviral medication was administered to 13 patients $(68 \%)$. Cidofovir was the most common antiviral medication given, with administration to 10 patients (53\%). Two of those patients were changed from cidofovir to the liposomal formulation and one patient was changed to the enteral formulation, brincidofovir. Other antiviral medications given either for prophylaxis or treatment included acyclovir given to five patients (26\%), ganciclovir given to three patients (16\%), valacyclovir given to two patients (11\%), foscarnet given to two patients (11\%), and ribavirin given to one patient (5\%).

We compared patient characteristics and clinical features between nonsurvivors and survivors (Table 3). Patient characteristics that were associated with mortality were the cause of immunocompromised state $(p=0.015)$. Clinical characteristics associated with mortality included meeting diagnostic criteria for MODS $(p=0.001)$, requiring RRT $(p=0.01)$, PIM3 predicted mortality $(p=0.011)$, and treatment with cidofovir $(p=0.005)$.

We performed a matched case control analysis of immunocompromised cases and immunocompetent controls with severe adenoviral respiratory infection (Table 4). All 19 controls were diagnosed by respiratory viral PCR. There were no differences in age, gender, or PIM3 predicted mortality between cases and controls. There were no differences in hospital or ICU lengths of stay or rates of respiratory failure. As compared to the immunocompetent controls, the immunocompromised cases were more likely to have MODS $(p=0.01)$, require RRT $(p=0.02)$, have lower $\operatorname{ALC}(p=$ $0.004)$, and not survive to discharge $(p=0.004)$.

Long-term respiratory morbidity was assessed one year after adenovirus infection in survivors. Of the eight immunocompromised patients who survived to discharge, one died three months later. This left seven patients included in the follow-up assessment. Five patients (71\%) had at least one chronic respiratory diagnosis. Four of the patients (57\%) were prescribed daily inhaled steroid medication. Oxygen supplementation was required in three patients $(43 \%)$ and three patients (43\%) required chronic mechanical ventilation. Of the 18 surviving control patients, one died two months later from an unrelated illness and six were lost to followup, leaving 11 patients for analysis. One year after severe respiratory adenoviral infection, three $(27 \%)$ of the control patients were on chronic mechanical ventilation, though all three were on mechanical ventilation prior to their adenoviral infection. Six of the control patients (55\%) were on daily inhaled respiratory medications, and four (36\%) had no respiratory diagnosis nor required any respiratory medications.

\section{Discussion}

This multicenter study found that severe adenoviral respiratory infection in the immunocompromised population has high associated morbidity and case fatality. We found a longer ICU length of stay (20 days, IQR 7 to 48) and length of mechanical ventilation (12 days, IQR 1-27 days) than previously reported for critically ill pediatric HSCT patients (ICU LOS 3 days [25] and mechanical ventilation 6 days [26]). Our reported case fatality rate of $58 \%$ is one of the higher rates reported for adenoviral infection in the immunocompromised population, supporting suggestions that have been made in prior studies that respiratory adenoviral infection has higher associated mortality than other forms of adenoviral infection in the immunocompromised population $[15,16,27]$. Comparison of our immunocompromised cohort with a matched control group of immunocompetent patients further highlights the high morbidity and mortality for immunocompromised patients with severe respiratory 


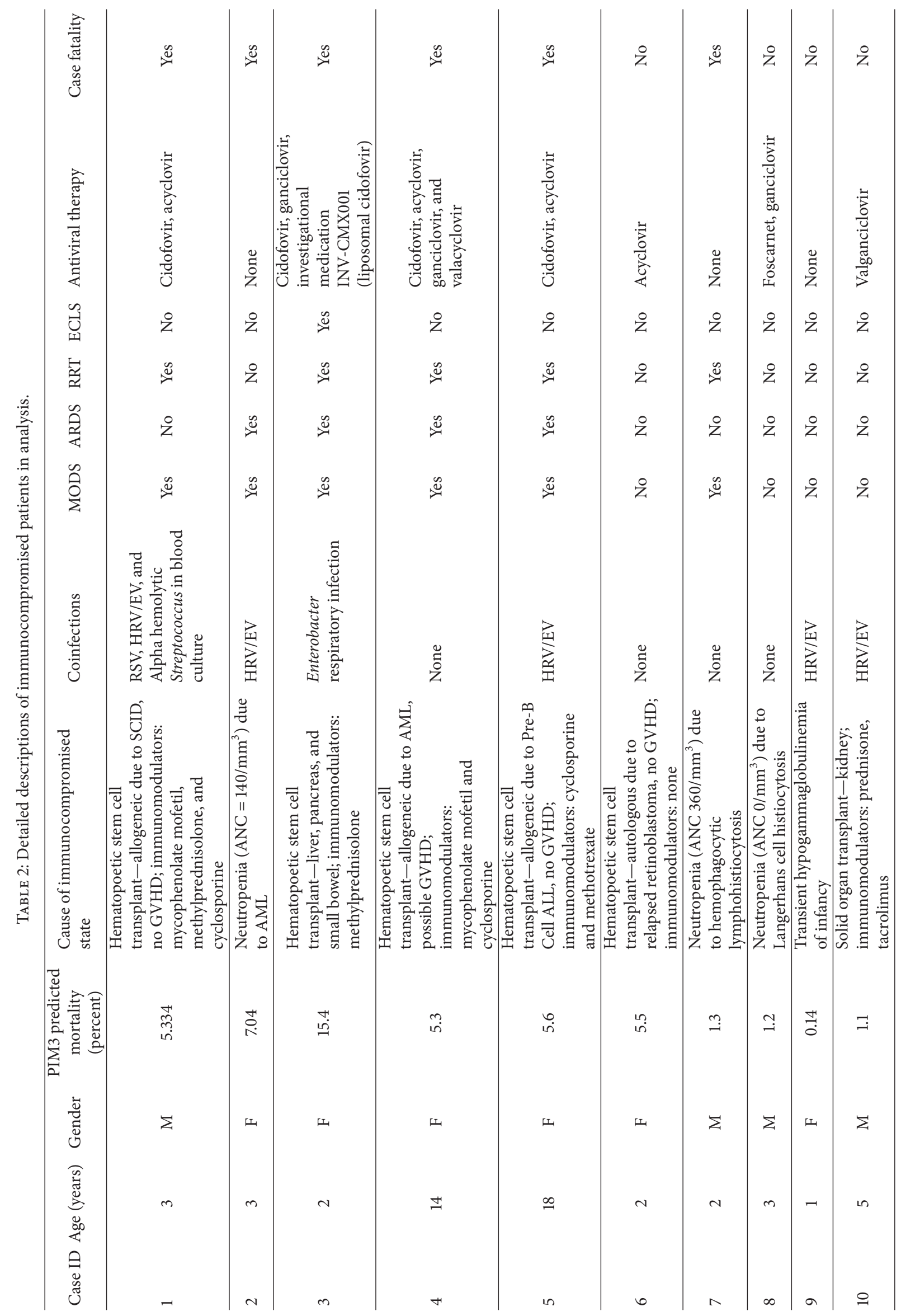




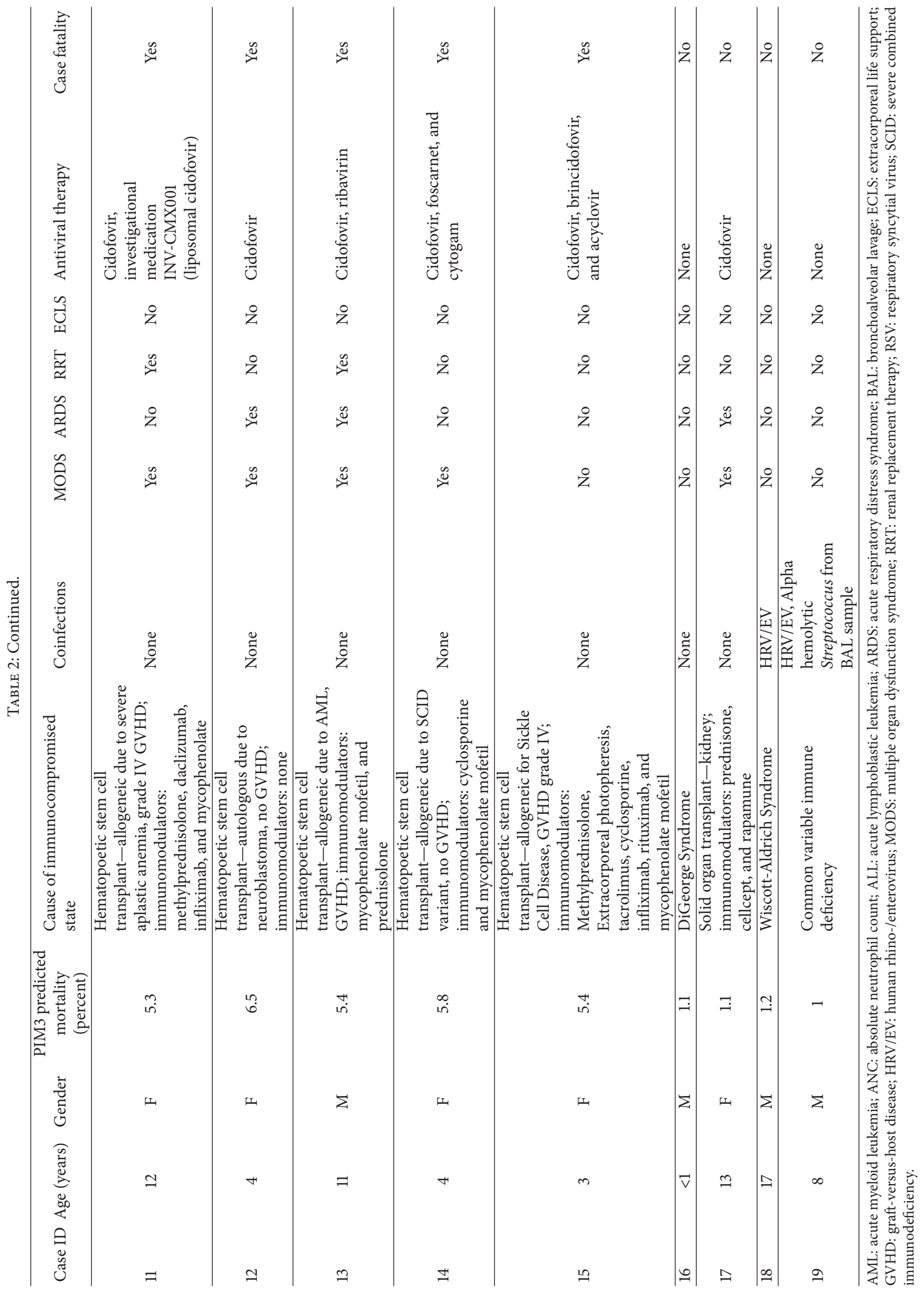


TABLE 3: Patient and clinical characteristics stratified by survival.

\begin{tabular}{|c|c|c|c|}
\hline Characteristic & $\begin{array}{c}\text { Nonsurvivors } \\
\quad(n=11)\end{array}$ & $\begin{array}{l}\text { Survivors } \\
(n=8)\end{array}$ & $p$ value \\
\hline Age (years) & $3.9(3.0-12.7)$ & $3.9(1.8-11.2)$ & 0.56 \\
\hline Female gender, $n(\%)$ & $8(72 \%)$ & $3(38 \%)$ & 0.18 \\
\hline Cause of immunocompromised state, $n(\%)$ & & & 0.015 \\
\hline Solid organ transplant & $1(13 \%)$ & $2(25 \%)$ & \\
\hline Blood and marrow transplant & $8(73 \%)$ & $1(13 \%)$ & \\
\hline Human immunodeficiency virus & 0 & 0 & \\
\hline Neutropenia & $2(28 \%)$ & $1(13 \%)$ & \\
\hline Congenital immunodeficiency & 0 & $4(50 \%)$ & \\
\hline Hospital LOS (days) & $46(17-145)$ & $45(19-88)$ & 0.90 \\
\hline ICU LOS (days) & $19(7-48)$ & $24(7-46)$ & 0.80 \\
\hline Mechanical ventilation & $11(100 \%)$ & $6(75 \%)$ & 0.16 \\
\hline Ventilator days & $14(5-34)$ & $5(0-21)$ & 0.17 \\
\hline Acute respiratory distress syndrome & $6(55 \%)$ & $1(13 \%)$ & 0.15 \\
\hline Multiple organ dysfunction syndrome & $10(91 \%)$ & $1(13 \%)$ & 0.001 \\
\hline Renal replacement therapy & $7(64 \%)$ & 0 & 0.01 \\
\hline Extracorporeal life support & $1(13 \%)$ & 0 & 0.58 \\
\hline PIM3 predicted mortality (percent) & $5.6(5.3-6.5)$ & $1.1(1.1-3.3)$ & 0.011 \\
\hline Antiviral treatment & $9(82 \%)$ & $4(50 \%)$ & 0.32 \\
\hline Treatment with cidofovir & $9(82 \%)$ & $1(13 \%)$ & 0.005 \\
\hline Coinfection & $4(36 \%)$ & $4(50 \%)$ & 0.66 \\
\hline Viral coinfection & $4(36 \%)$ & $3(38 \%)$ & 0.38 \\
\hline Bacterial coinfection & $2(18 \%)$ & $1(13 \%)$ & 1 \\
\hline Absolute lymphocyte count (cells $/ \mathrm{mm}^{3}$ ) & $480(40-650)$ & $1150(459-4070)$ & 0.13 \\
\hline
\end{tabular}

Values expressed as $n$ (\%). Continuous variables are shown as median (interquartile range). ICU: intensive care unit; LOS: length of stay; PIM3: Paediatric Index of Mortality 3.

TABLE 4: Matched case control analysis of immunocompromised cases with severe adenoviral respiratory infection and immunocompetent controls with severe adenoviral respiratory infection.

\begin{tabular}{|c|c|c|c|}
\hline Characteristic & Immunocompromised cases $(n=19)$ & Immunocompetent controls $(n=19)$ & $p$ value \\
\hline Age (years) & $3.9(\mathrm{IQR} 2.6-12.7)$ & $3.6($ IQR $1-8.5)$ & 0.11 \\
\hline Female sex & $11(58 \%)$ & $12(63 \%)$ & 0.74 \\
\hline PIM3 predicted mortality (percent) & 5.3 (IQR 1.1-5.6) & 3.3 (IQR 1.1-4.6) & 0.13 \\
\hline Hospital LOS (days) & 45 (IQR 17-112) & 13 (IQR 4-34) & 0.06 \\
\hline ICU LOS (days) & 20 (IQR 7-48) & 6 (IQR 3-23) & 0.07 \\
\hline Respiratory failure & $17(89 \%)$ & $14(74 \%)$ & 0.45 \\
\hline $\operatorname{ALC}\left(\right.$ cells $\left./ \mathrm{mm}^{3}\right)$ & 560 (IQR 50-1520) & 2490 (IQR 1840-4070) & 0.004 \\
\hline Bacterial coinfection & $3(5 \%)$ & $2(11 \%)$ & 1.00 \\
\hline Viral coinfection & $7(37 \%)$ & $4(21 \%)$ & 0.45 \\
\hline MODS & $11(58 \%)$ & $2(11 \%)$ & 0.01 \\
\hline ARDS & $7(37 \%)$ & $2(11 \%)$ & 1.00 \\
\hline RRT & $7(37 \%)$ & 0 & 0.02 \\
\hline ECLS & $1(5 \%)$ & 0 & 1.00 \\
\hline Case fatality & $11(58 \%)$ & $1(5 \%)$ & 0.004 \\
\hline
\end{tabular}

Values expressed as $n$ (\%). Continuous variables are shown as median (interquartile range). PIM3: Paediatric Index of Mortality 3; ICU: intensive care unit; LOS: length of stay; ALC: absolute lymphocyte count; MODS: multiple organ dysfunction syndrome; ARDS: acute respiratory distress syndrome; RRT: renal replacement therapy; ECLS: extracorporeal life support. 
adenoviral infection. The immunocompromised group had more MODS, received more renal replacement therapy, and had higher case fatality (58\% compared to $5 \%$ ).

We found an association between cause of immunocompromised state and case fatality, with the case fatality for the HSCT patients being very high (89\%). Similarly, de Mezerville et al. found higher mortality rates for pediatric HSCT patients with adenoviral infection as compared to SOT patients [16]. It is likely that the worse outcomes for the HSCT patients are related to their increased degree of compromised immune function.

Cidofovir is a broad-spectrum antiviral agent that inhibits DNA polymerase to which all adenovirus serotypes have been shown to be susceptible in vitro. Recently, cidofovir has been increasingly used in an off-label manner in the treatment of adenovirus in the immunocompromised population, particularly the HSCT population. Case reports and studies have documented successful treatment of adenoviral infection in the SOT and HSCT populations [14, 28, 29]. Conversely, our study found an association between treatment with cidofovir and mortality. This association is likely related to the severity of illness; patients who were more ill were more likely to be treated with cidofovir than those who were less ill.

A high number of patients in our study had coinfections $(42 \%)$, with the majority being other respiratory viral coinfections. Several other studies have found high adenoviral coinfection rates, ranging from 20 to $50 \%[2,21,30]$. The detection of additional viruses on respiratory viral PCR is potentially confounding in patients with severe viral respiratory illness as it may reflect active infection of multiple viruses or infection with a single virus and detection of viral shedding from a second virus. Human rhinoviruses and enteroviruses, which were the most common viruses codetected, continue to be shed for up to six weeks following active infection in previously healthy children [30,31]. We did not observe any association between coinfection and case fatality.

Consistent with the published literature, we found longterm respiratory morbidity to be high after respiratory adenovirus infection. Our findings that $55 \%$ of surviving immunocompetent and 57\% of surviving immunocompromised patients were using daily inhaled respiratory medications one year after adenoviral infection were lower than the $95 \%$ using inhaled corticosteroids five years after infection reported in one study [13]. However, our finding that $27 \%$ of surviving immunocompetent and $43 \%$ of surviving immunocompromised patients utilized supplemental oxygen at one year is higher than the $5-13 \%$ seen at long-term followup in previous studies $[12,13]$. Additionally, our finding that $27 \%$ of surviving immunocompetent and $43 \%$ of surviving immunocompromised patients required chronic mechanical ventilator support is novel. These findings suggest that patients with severe respiratory adenoviral infection are at high risk for long-term respiratory morbidity and that being immunocompromised may place patients at even higher risk.

This study was limited by its retrospective design and relatively small number of patients. Additionally, three methods of testing for adenovirus were used with varying sensitivity and specificity for adenovirus and other viruses causing coinfection. Although we found no association between coinfections and case fatality there was a high level of coinfections found in this study. This high coinfection rate, as well as the high morbidity in this critically ill population, makes it difficult to fully assess risk factors and confounding comorbidities. Finally, there was heterogeneity in the population studied with variability in patients based on location and services available. A prospective study with a larger number of centers would be able to better delineate risk factors.

\section{Conclusions}

Morbidity and case fatality associated with severe respiratory adenovirus infection in the immunocompromised population is high. This morbidity and case fatality appears to be higher than that of other sites of adenoviral infection and was particularly high in the HSCT population. Respiratory morbidity in survivors one year following adenoviral respiratory infection was considerable.

\section{Competing Interests}

The authors have no competing interests to report.

\section{Acknowledgments}

All work pertinent to this paper was conducted at the Children's National Medical Center, Brenner Children's Hospital, Hackensack University Medical Center, and the University of Maryland Medical Center.

\section{References}

[1] E. G. Rhee and D. H. Barouch, "Adenoviruses," in Principles and Practice of Infectious Diseases, M. Mandell, J. E. Bennett, and R. Dolin, Eds., pp. 2027-2033, Churchill Livingstone, Elsevier, Philadelphia, Pa, USA, 7th edition, 2010.

[2] M.-P. Lu, L.-Y. Ma, Q. Zheng, L.-L. Dong, and Z.-M. Chen, "Clinical characteristics of adenovirus associated lower respiratory tract infection in children," World Journal of Pediatrics, vol. 9, no. 4, pp. 346-349, 2013.

[3] S. Alharbi, P. Van Caeseele, R. Consunji-Araneta et al., "Epidemiology of severe pediatric adenovirus lower respiratory tract infections in Manitoba, Canada, 1991-2005," BMC Infectious Diseases, vol. 12, article 55, 2012.

[4] M. Sakamoto, N. Yazaki, N. Katsushima, K. Mizuta, H. Suzuki, and Y. Numazaki, "Longitudinal investigation of epidemiologic feature of adenovirus infections in acute respiratory illnesses among children in Yamagata, Japan (1986-1991)," Tohoku Journal of Experimental Medicine, vol. 175, no. 3, pp. 185-193, 1995.

[5] Q. Sun, W. Jiang, Z. Chen et al., "Epidemiology and clinical features of respiratory adenoviral infections in children," European Journal of Pediatrics, vol. 173, no. 4, pp. 441-444, 2014.

[6] P. Gupta, J. D. Tobias, S. Goyal et al., "Prolonged mechanical support in children with severe adenoviral infections: a case series and review of the literature," Journal of Intensive Care Medicine, vol. 26, no. 4, pp. 267-272, 2011.

[7] P. Prodhan, A. T. Bhutta, J. M. Gossett et al., "Extracorporeal membrane oxygenation support among children with adenovirus infection: a review of the extracorporeal life support 
organization registry," ASAIO Journal, vol. 60, no. 1, pp. 49-56, 2014.

[8] D. M. O. Becroft, "Sequelae of adenovirus type 21 infection in young children," Journal of Clinical Pathology, vol. 24, no. 1, pp. 72-82, 1971.

[9] W. M. Wenman, R. D. Pagtakhan, M. H. Reed, V. Chernick, and W. Albritton, "Adenovirus bronchiolitis in Manitoba. Epidemiologic, clinical, and radiologic features," Chest, vol. 81, no. 5, pp. 605-609, 1982.

[10] Z. Callaway, S. H. Kim, J. Y. Kim et al., "Adenovirus infection with serious pulmonary sequelae in Korean children," The Clinical Respiratory Journal, vol. 5, no. 2, pp. 92-98, 2011.

[11] L. Spigelblatt and R. Rosenfeld, "Hyperlucent lung: long-term complication of adenovirus type 7 pneumonia," Canadian Medical Association Journal, vol. 128, no. 1, pp. 47-49, 1983.

[12] P. Murtagh, V. Giubergia, D. Viale, G. Bauer, and H. G. Pena, "Lower respiratory infections by adenovirus in Children. Clinical features and risk factors for bronchiolitis obliterans and mortality," Pediatric Pulmonology, vol. 44, no. 5, pp. 450-456, 2009.

[13] J. A. Castro-Rodriguez, C. Daszenies, M. Garcia, R. Meyer, and R. Gonzales, "Adenovirus pneumonia in infants and factors for developing bronchiolitis obliterans: a 5-year follow-up," Pediatric Pulmonology, vol. 41, no. 10, pp. 947-953, 2006.

[14] U. Yusuf, G. A. Hale, J. Carr et al., "Cidofovir for the treatment of adenoviral infection in pediatric hematopoietic stem cell transplant patients," Transplantation, vol. 81, no. 10, pp. 13981404, 2006.

[15] A. M. La Rosa, R. E. Champlin, N. Mirza et al., "Adenovirus infections in adult recipients of blood and marrow transplants," Clinical Infectious Diseases, vol. 32, no. 6, pp. 871-876, 2001.

[16] M. H. de Mezerville, R. Tellier, S. Richardson, D. Hébert, J. Doyle, and U. Allen, "Adenoviral infections in pediatric transplant recipients: a hospital-based study," Pediatric Infectious Disease Journal, vol. 25, no. 9, pp. 815-818, 2006.

[17] P. Bordigoni, A.-S. Carret, V. Venard, F. Witz, and A. L. Faou, "Treatment of adenovirus infections in patients undergoing allogeneic hematopoietic stem cell transplantation," Clinical Infectious Diseases, vol. 32, no. 9, pp. 1290-1297, 2001.

[18] V. Venard, A.-S. Carret, D. Corsaro, P. Bordigoni, and A. Le Faou, "Genotyping of adenoviruses isolated in an outbreak in a bone marrow transplant unit shows that diverse strains are involved," Journal of Hospital Infection, vol. 44, no. 1, pp. 71-74, 2000.

[19] M. G. Michaels, M. Green, E. R. Wald, and T. E. Starzl, "Adenovirus infection in pediatric liver transplant recipients," Journal of Infectious Diseases, vol. 165, no. 1, pp. 170-174, 1992.

[20] M. S. Zinter, C. C. Dvorak, A. Spicer, M. J. Cowan, and A. Sapru, "New insights into multicenter PICU mortality among pediatric hematopoietic stem cell transplant patients," Critical Care Medicine, vol. 43, no. 9, pp. 1986-1994, 2015.

[21] M. C. Spaeder, "Severe adenoviral respiratory infection in children," Intensive Care Medicine, vol. 39, no. 6, pp. 1157-1158, 2013.

[22] B. Goldstein, B. Giroir, and A. Randolph, "International pediatric sepsis consensus conference: definitions for sepsis and organ dysfunction in pediatrics," Pediatric Critical Care Medicine, vol. 6, no. 1, pp. 2-8, 2005.

[23] T. B. Definition, "Acute respiratory distress syndrome," The Journal of the American Medical Association, vol. 307, no. 23, pp. 2526-2533, 2012.
[24] L. Straney, A. Clements, R. C. Parslow et al., "Paediatric index of mortality 3: an updated model for predicting mortality in pediatric intensive care," Pediatric Critical Care Medicine, vol. 14, no. 7, pp. 673-681, 2013.

[25] S. J. Jacobe, A. Hassan, P. Veys, and Q. Mok, "Outcome of children requiring admission to an intensive care unit after bone marrow transplantation," Critical Care Medicine, vol. 31, no. 5, pp. 1299-1305, 2003.

[26] J. P. J. van Gestel, M. B. Bierings, S. Dauger et al., "Outcome of invasive mechanical ventilation after pediatric allogeneic hematopoietic SCT: results from a prospective, multicenter registry," Bone Marrow Transplantation, vol. 49, no. 10, pp. 12871292, 2014.

[27] A. Baldwin, H. Kingman, M. Darville et al., "Outcome and clinical course of 100 patients with adenovirus infection following bone marrow transplantation," Bone Marrow Transplantation, vol. 26, no. 12, pp. 1333-1338, 2000.

[28] F. Legrand, D. Berrebi, N. Houhou et al., "Early diagnosis of adenovirus infection and treatment with cidofovir after bone marrow transplantation in children," Bone Marrow Transplantation, vol. 27, no. 6, pp. 621-626, 2001.

[29] M. E. Mateos, E. López-Laso, J. L. Pérez-Navero, M. J. Peña, and M. J. Velasco, "Successful response to cidofovir of adenovirus hepatitis during chemotherapy in a child with hepatoblastoma," Journal of Pediatric Hematology/Oncology, vol. 34, no. 7, pp. e298-e300, 2012.

[30] S. Berciaud, F. Rayne, S. Kassab et al., "Adenovirus infections in Bordeaux University Hospital 2008-2010: clinical and virological features," Journal of Clinical Virology, vol. 54, no. 4, pp. 302-307, 2012.

[31] T. Jartti, P. Lehtinen, T. Vuorinen, M. Koskenvuo, and O. Ruuskanen, "Persistence of rhinovirus and enterovirus RNA after acute respiratory illness in children," Journal of Medical Virology, vol. 72, no. 4, pp. 695-699, 2004. 


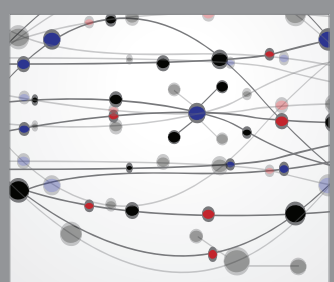

The Scientific World Journal
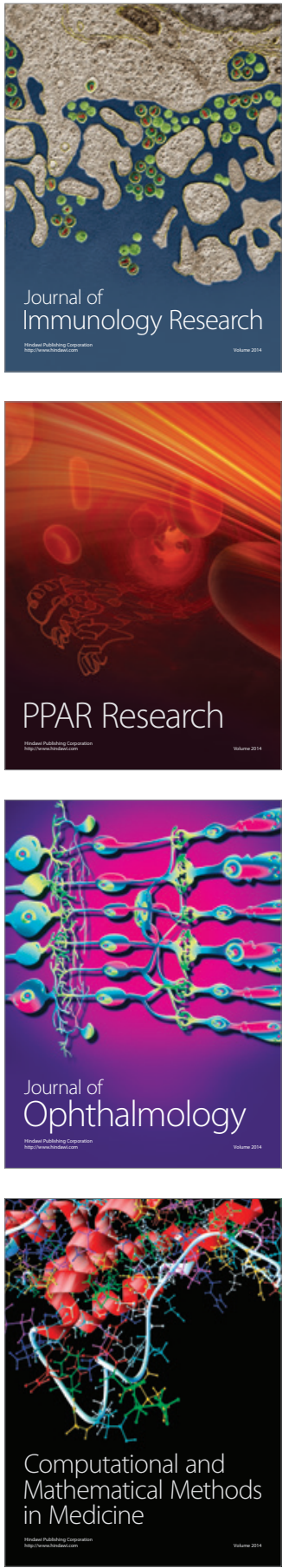

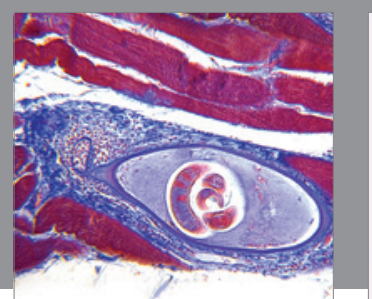

Gastroenterology Research and Practice

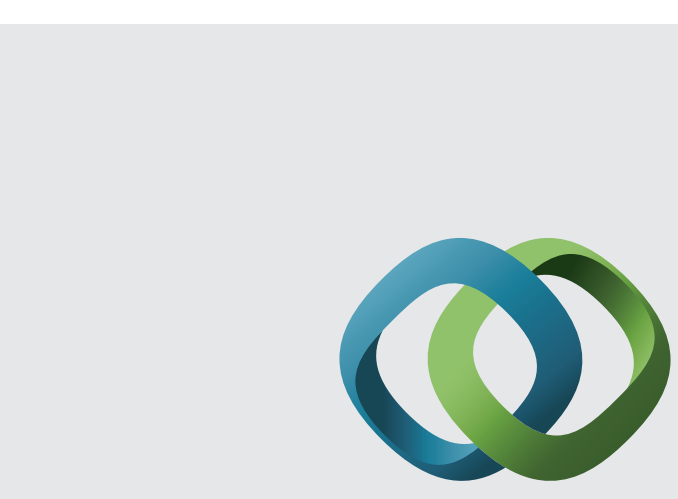

\section{Hindawi}

Submit your manuscripts at

http://www.hindawi.com
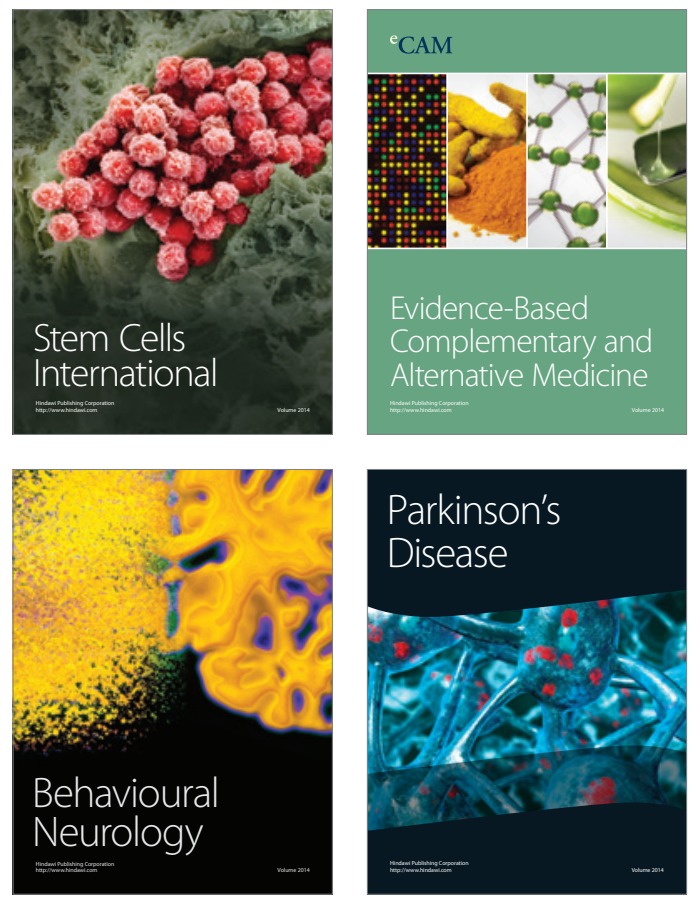
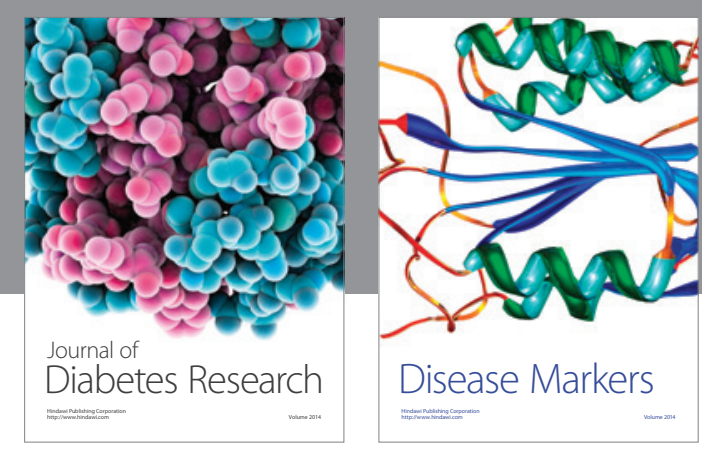

Disease Markers
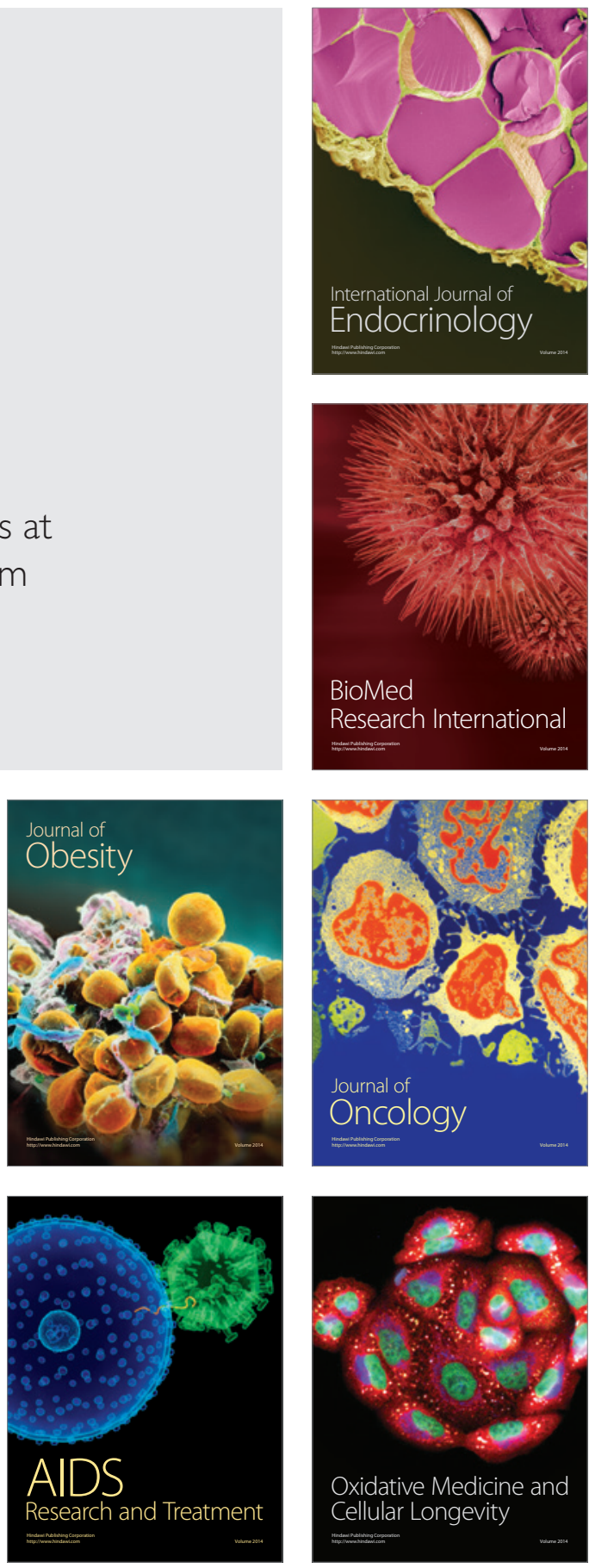